\title{
Prediction of the 4D Chromosome Structure From Time-Series Hi-C Data
}

\author{
Max Highsmith, Jianlin Cheng \\ Department of Electrical Engineering and Computer Science, University of Missouri, Columbia, \\ MO 65211, USA
}

\section{Abstract}

Chromatin conformation plays an important role in a variety of genomic processes. $\mathrm{Hi}-\mathrm{C}$ Data is frequently used to analyse structural features of chromatin such as $A B$ compartments, topologically associated domains, and 3D structural models. Recently the genomics community has displayed growing interest in chromatin dynamics over time. Here we present 4DMax, a novel method which uses time-series $\mathrm{Hi}-\mathrm{C}$ data to predict dynamic chromosome conformation. Using both synthetic data and real time-series $\mathrm{Hi}-\mathrm{C}$ data from processes such as induced pluripotent stem cell reprogramming and cardiomyocyte differentiation, we construct fluid four dimensional models of individual chromosomes. These predicted 4D models effectively interpolate chromatin position across time, permitting prediction of unknown Hi-C contact maps at intermittent time points. Our results demonstrate that 4DMax correctly recovers higher order features of chromatin such as $A B$ compartments and topologically associated domains, even at time points where $\mathrm{Hi}-\mathrm{C}$ data is not made available to the algorithm. Use of 4DMax may alleviate the cost of expensive $\mathrm{Hi}-\mathrm{C}$ experiments by interpolating intermediary timepoints while also providing valuable visualization of dynamic chromatin changes.

\section{Background}

The three-dimensional (3D) conformation of the genome has been shown to play an important role in a variety of genomic processes such as gene expression, gene replication and gene methylation ${ }^{1,2} 3$. Various techniques have developed for the analysis of three dimensional genome conformation, one of the most prominent being $\mathrm{Hi}-\mathrm{C}^{12}$, an improvement of the chromosome conformation captured (3C) technology. Hi-C data can be used to examine a plethora of higher order structural features such as: $A B$ compartments ${ }^{1}$, topological associated domains (TADs) ${ }^{4}$ and 3D structural models ${ }^{6}$.

As genomic sequencing has become cheaper more researchers have begun to generate time-series $\mathrm{Hi}-\mathrm{C}$ data ${ }^{7,8}$. In such datasets $\mathrm{Hi}-\mathrm{C}$ contact maps are obtained at multiple points in a time dependent genetic process. Some of these biological processes include: Induced stem cell pluripotency ${ }^{7}$ and cardiomyocyte differentiation ${ }^{8}$. While a plethora of meaningful and interesting observations have already been extracted from these datasets, analysis has been 
primarily constrained to comparing and contrasting individual points in the time series rather than the comprehensive analysis of four dimensional (4D) chromatin conformation changes over multiple time points (i.e. three dimensional conformation plus the 4 th dimension of time). The need for novel 4D analysis has been identified as a critical and emerging area of research ${ }^{9}$.

To address this need we introduce 4DMax, a maximum-likelihood based algorithm for predicting the transformation of chromatin conformation over the 4th dimension (time). By using spatial restraints derived from $\mathrm{Hi}-\mathrm{C}$ contact matrices we provide a tool which permits the generation of a predictive $4 \mathrm{D}$ video of chromatin conformational changes throughout the time series. 4DMax can be used to interpolate higher order chromatin features at times where no data is available while also providing valuable visualizations of chromosomal processes.

To date, only one other published computational method for modeling 4D transitions of chromatin exists, TADdyn ${ }^{10}$. Our 4DMax algorithm differs from TADdyn in 3 key ways.

Firstly, we utilize gradient descent optimization ${ }^{11}$ of a spatial restraint based maximum-likelihood function ${ }^{12,13}$ whereas the TADDyn approach utilizes monte carlo based simulated annealing.

Secondly, TADyn focuses on small $\sim 2 M B$ segments of the genome with emphasis on transcriptional dynamics while our algorithm provides models of entire chromosomes. Our broader scope permits meaningful analysis of higher level structures such as TADs and AB compartments across time.

Thirdly, we demonstrate that 4DMax can use generated models as an interpolation mechanism for predicting chromosomal contact maps at time points for which no $\mathrm{Hi}-\mathrm{C}$ data has been gathered.

The value of 4DMax is demonstrated through the construction of 4D models using contact maps derived from a mean-field simulated chromosomal looping process as well as multiple real time series $\mathrm{Hi}-\mathrm{C}$ datasets. By studying the interrelation between contact maps we are capable of identifying meaningful characteristics of the genomic process unavailable from analysis of only individual timepoints. We successfully recover higher order conformational information such as $A B$ compartments from the predicted 4D structure, even at time points where true Hi-C maps are intentionally excluded. Out of the box 4DMax can be easily inserted into any analytic pipeline focused on time-series $\mathrm{Hi}-\mathrm{C}$ analysis.

\section{Results}

\section{Overview of 4DMax approach.}

In Figure 1 we outline the overall framework of 4DMax. First we gather intrachromosomal contact matrices from different time points in a genomic process. Next we convert contact matrices into spatial restraints using the $D=I F^{\gamma}$ equation used frequently in 3D modeling literature ${ }^{6}$. We then assign a parameter, granularity, to denote the number of temporal snapshots where the spatial position of our 4D model will be identified. Then, using a maximum likelihood approach from probability theory, we define a likelihood function which measures the agreement of our structures position at each time point with temporally adjacent spatial 
restraints. We then initiate an unfolded structure and incrementally adjust its position to maximize our likelihood function using a gradient ascent algorithm. After training, a smooth 4Dmodel is created which can be visualized in movie format. From this 4DModel we extract synthetic $\mathrm{Hi}-\mathrm{C}$ contact maps at time points of interest. We then use these extracted $\mathrm{Hi}-\mathrm{C}$ contact maps for downstream Hi-C analysis such as $\mathrm{AB}$ compartment classification and topologically associated domain (TAD) identification.

\section{DMax correctly reconstructs models of synthetic time series Hi-C data.}

We first created a simple, hypothetical chromosome and developed two theoretical structural progressions for the changing conformation of this chromosome. Both simulations are composed of 11 chromosomal bins and evolve over a 6 day process. Each 4D structure begins and ends identically, the initial chromatin state being in a looped formation and the final state being fully elongated. The two structures differ in their respective paths taken from their initial and final states. In structure 1 the loop unravels as if pulled on both ends while in structure 2 the loop swings open (figure 1a). As a consequence of these differences in paths, on day 3 , there is strong interaction between bins 4 and 6 on structure 1 but no such interaction exists on structure 2.

We first define contact maps for each of the 6 time points on both structures (Supplementary Figures 1) and use these contacts as inputs to 4DMax to generate novel 4D structures (Supplementary Videos 1). We then simulate Hi-C experiments at the 6 time points using the generated structure and obtain contact maps with above .95 pearson correlation (PCC) with corresponding input contact maps (Supplementary Figure 2). Furthermore, visual inspection of the two generated videos accurately display the unique behaviors of unraveling and swinging open previously described (Supplementary Videos 1).

We test the effectiveness of 4DMax in capturing 4D movement and predicting 3D position at time points where contact map information is unavailable. We run four experiments for each synthetic structure excluding contact maps for days 1,2,3,4 respectively. The PCC values between original synthetic $\mathrm{Hi}-\mathrm{C}$ maps and their corresponding interpolations remain high ranging from 0.82-0.99 (Supplementary Figure 2). Visually, we continue to observe the expected unraveling and swinging behaviors in each $4 \mathrm{~d}$ video, even with excluded data (Supplementary Videos 1).

\section{DMax predicts fluid 4D models of induced pluripotent stem cell differentiation in mice.}

We apply 4DMax to a 10 day time series $\mathrm{Hi}-\mathrm{C}$ dataset of induced stem cell pluripotency in mice ${ }^{10}$. We use intrachromosomal Hi-C contact maps from day 0 (Beta), 2, 4, 6, 8 and 10 (PSC). We select a granularity of 21 , ensuring that each time point for which real data is available occurs within the time interval partition. We demonstrate that results remain rigid with other granularities (fig2a). 4DMax successfully produces smoothly changing structures for each chromosome (Fig2c, Supplementary videos 2). We frequently observe a decrease in compression of 4D models as the induced pluripotency process progresses (Fig2c, Supplementary videos 2). The 4DMax predictions for chromosomal position at the input times shows high similarity to 3D structures generated by previously built state of the art 3D modeling algorithms with average $\mathrm{SPC}=0.76$ and $\mathrm{PCC}=0.75^{13}$ (Supplementary Figures 3). 
Using the 4DMax predictions we then simulated Hi-C experiments (fig2d, supplementary Figures 4) at each of the input time points to obtain synthetic Hi-C maps. We compare these synthetic maps to their corresponding real contact maps and observe high SPC values ranging (0.53-0.82). These values are consistently higher than the similarities seen between contact maps on Days 0 and 10 (0.46-0.68) (Supplementary Figures 5).

\section{DMax predicts fluid 4D models of cardiomyocyte differentiation in humans.}

To verify the effectiveness of varied $\mathrm{Hi}-\mathrm{C}$ datasets we also apply 4DMax to a 14 day time series $\mathrm{Hi}-\mathrm{C}$ dataset of cardiomyocyte cell differentiation ${ }^{8}$. The cardiomyocytes dataset contains $\mathrm{Hi}-\mathrm{C}$ contact maps assayed at irregularly timed intervals on days: $0,2,5$ and 14 . We build 4DModels with a granularity of 15 , ensuring that each time point for which real data is available occurs within the time interval partition., preserving the uneven timing of the contact maps. (Supplementary Figures 6). 4DMax again produces fluidly changing 4D models.

(Supplementary videos 3) We then simulate Hi-C experiments to obtain synthetic contact maps from the 4D model at the 4 input times and observe SPC values ranging from 0.54 to 0.92 between synthetic maps and their correspondingly timed real Hi-C data. (Supplementary Figures 7). We compare 4DMax reconstructed contact maps to real contact maps across all permutations of input times and observe SPC values are highest with corresponding times in $93.2 \%$ of the reconstructions, indicating the high correlation between real and reconstructed maps is significant relative to other Hi-C contact maps.

\section{Interpolation of Time Series Hi-C Data using 4DMax generated models show high consistency with experimental Hi-C.}

To evaluate the rigidity of 4DMax in its prediction of chromosomal position at timepoints between available contact maps we ran 4 experiments on each chromosome where we generated 4D models of the iPSC dataset while excluding $\mathrm{Hi}-\mathrm{C}$ data for individual timepoints: D2, D4, D6, D8. We call these models the "iPSC Interp models". The iPSC Interp models show high similarity to 4D models generated by the complete iPSC dataset (SPC>.99, in all chromosomes besides 1,4,5 PCC>.96), indicating the algorithm's resilience to missing Hi-C data (Supplementary Figure 14, Supplementary Videos 4). We then ran synthetic Hi-C experiments on the iPSC interp models at the time point for which their data was excluded to obtain interpolated contact maps. We compare these interpolated contact maps to corresponding real $\mathrm{Hi}-\mathrm{C}$ contact maps and find high correlation with mean $\mathrm{SPC}=0.73$ with values ranging from $0.62-0.80$ (Fig3a). In $24 \%$ of the experiments our interpolated contact maps show higher correlation to the real Hi-C contact maps than their biological replicate (Fig3b, Supplementary Figures 10). These results indicate that 4DMax is effective at predicting intermittent structures for time points where no $\mathrm{Hi}-\mathrm{C}$ data is available.

We also perform interpolation experiments using the cardiomyocyte dataset where we exclude Hi-C input data on day 2. We refer to the resultant 4Dmodesl as the "Cardio Interp models". The Cardio Interp models show high correlation to 4D models generated using the complete cardiomyocyte dataset (Supplementary Figures 15, Supplementary Videos 5). We obtain synthetic Hi-C contact maps on day 2 from the Cardio Interp Model and compare these 
interpolation maps to the real day $2 \mathrm{Hi}-\mathrm{C}$ contact maps and find SPC values ranging from $0.57-0.87$ (Fig3c). In 6 of the chromosomes (28\%) our interpolation shows higher correlation to the real $\mathrm{Hi}-\mathrm{C}$ map than a biological replicate (Fig3d, Supplementary Figures 8 ). These results indicate versatility in the time-series datasets for which our 4DMax algorithm can effectively interpolate Hi-C data.

\section{DMax correctly preserves and predicts $A B$ compartment assignment.}

A primary value of $\mathrm{Hi}-\mathrm{C}$ data is its utility in illuminating higher order structural features of chromatin. One of the most prolific of these structural features are megabase scale subnuclear compartments called $A B$ compartments. Regions of the genome are assigned to either compartment $A$ or compartment $B$ where the $A$ compartment is associated with gene activity and euchromatin while the $B$ compartment is associated with inactive, heterochromatin. $A B$ compartment assignment can be derived by principal component analysis (PCA) of pearson correlation matrices derived from Hi-C contact maps (Methods). We first perform comparative $\mathrm{AB}$ compartment analysis on real $\mathrm{Hi}-\mathrm{C}$ contact maps, and contact maps reconstructed from iPSC full models. We observe high visual similarity between pearson correlation matrices of reconstructed and corresponding real $\mathrm{Hi}-\mathrm{C}$ data across all chromosomes and timepoints (fig3a, Supplementary figures 18).

The iPSC dataset has previously been shown to undergo pronounced changes to compartmental organization as time progresses. Visually we observe high similarity between Reconstructed and Real AB compartment vectors at each point in the time series (fig3b, Supplementary Figures 13). We quantify this progression by treating ab compartment vectors as input vectors to PCA to obtain trajectory curves for each chromosome (fig 3c, Supplementary Figures 11). The trajectories of real and reconstructed compartments match one another closely. These analysis indicate that the 4D models generated by 4DMax maintain the higher order information needed for $A B$ compartment analysis.

We also compared the $A B$ compartment profiles of our interpolated iPSC matrices to $A B$ compartment profiles of real $\mathrm{Hi}-\mathrm{C}$ contact maps (fig3d, Supplementary Figures Fig 12). In all 4 models we see PCC values greater than 0.96. Furthermore, when comparing interpolated $A B$ compartment profiles to the $A B$ compartment profiles of real $\mathrm{Hi}-\mathrm{C}$ contact maps across all times in the iPSC process, we find the highest correlation at the interpolated timepoints (fig3d, Supplementary Figures Fig12). For example, we built an interpolation model for 4D chromatin structure excluding contact information on day 6 , instead only showing the algorithm contact information for days $0,2,4,8$ and 10. 4DMax then made predictions for the chromosomal conformation on day 6 . The output prediction for chromosomal conformations on day 6 were more similar to the real contact matrices on day $6(0.97)$ than they were to any of the contact maps the algorithm was exposed to $(0.93,0.95,0.96,0.88)$. This trend is consistent across all interpolation models and is crucial as it indicates that 4DMax is accurately predicting changes to $\mathrm{AB}$ compartment profiles, rather than simply obtaining high correlation due to maintained ab compartment profiles between adjacent timepoints. 


\section{DMax correctly preserves and predicts TAD border positioning.}

Another prolific use of $\mathrm{Hi}-\mathrm{C}$ data is the identification of topologically associated domains (TADs) ${ }^{5}$. We used the Hi-C analysis tool HiCtool to identify TADs from contact maps in the iPSC dataset. We then use HiCtool to identify TADs with synthetic contact maps derived from 4DMax reconstruction and interpolation models (Fig 5ab). We observe high similarity in TAD profiles of reconstructed synthetic maps and real $\mathrm{Hi}-\mathrm{C}$ contact maps with a mean percent overlap of $85 \%$ and a peak of $99 \%$ on chromosome 9 . We also observe high similarity in TAD profiles of interpolated synthetic maps and real $\mathrm{Hi}-\mathrm{C}$ contact maps with a mean percent overlap of $84 \%$ and a peak of $97 \%$ on chromosome 11 .

\section{DMax completes in tractable time for human and mouse chromosome construction.}

4DModel generation time is determined by three parameters: training epochs, granularity and bin quantity. Run time scales linearly with number of training epochs (supplementary figures 16). Empirically we observe 400 epochs as sufficient to obtain organized and consistent conformational changes in 4D models for both datasets (Supplementary videos 6). Granularity, defined as the number of tracked discrete time points in the interval, also impacts run time linearly. (supplementary figures 16). Bin quantity, defined as the number of discrete spatial points tracked per time point, is dependent on chromosomal length and resolution. We observe super linear growth of time as bin quantity increases (Figure $6 a)$. We are capable of computing 4DMoles of $500 \mathrm{~kb}$ resolution chromosomes in a matter of minutes and the largest $50 \mathrm{~kb}$ chromosomes in under 1.5 hours.

\section{DMax predictions remain stable against change in time point granularity.}

We compared the 4D structures of the same chromosomes in the cardiomyocyte dataset with varying granularity of: $15,29,43,57,71$. For each granularity comparison we used the average correlation between timepoints present in both structures Fig (6b, Supplementary videos 7). We see minimal discrepancies between our maximal and minimal granularity values with the average correlation $(\mathrm{PCC}=0.90 ; \mathrm{SPC}=0.94)$ reaching as high as $(\mathrm{PCC}=0.94, \mathrm{SPC}=0.99)$ on chromosome 9 . These results indicate stability to changes in granularity.

\section{DMax predictions remain stable to variation in $\mathrm{Hi}-\mathrm{C}$ contact matrix resolution.}

We compared the 4D structures of the same chromosomes in the iPSC dataset at $500 \mathrm{~kb}$ and $50 \mathrm{~kb}$ resolution. The structures from $50 \mathrm{~kb}$ resolution were reduced to $500 \mathrm{~kb}$ by averaging the position of every 10 consecutive spatial points $\mathrm{Fig}(6 \mathrm{c})$. The average correlation between structures remains high $(\mathrm{SPC}=0.84, \mathrm{PCC}=0.83)$ reaching $(\mathrm{SPC}=0.96, \mathrm{PCC}=0.97)$ for chromosome 8 (Supplementary Figures 19). This indicates consistent 4DModel predictions across varying resolutions. 


\section{Discussion}

Here we present 4DMax, a method used to examine time-dependent dynamics of chromatin during genomic processes. 4DMax is the second published tool to simulate structural changes to chromatin over time and is the first of its kind to provide comprehensive chromosome wide predictions of 4D dynamics. By converting contact maps at select times into spatial restraints, using these restraints to build a likelihood based objective function, and optimization with gradient ascent, 4DMax constructs fluid 4DModels.

We validate the effectiveness of 4DMax in predicting 4D conformations using both synthetic chromosomal conformations and real time-series $\mathrm{Hi}-\mathrm{C}$ datasets from mice and humans. From these visualizations we often observe pronounced changes to the positioning of chromosomes over time such as the progressive decompression of mice chromosomes as they become pluripotent. From our 4D models we visually observe the preservation of preferentially interacting regions such of TADs, providing valuable visual representations of how such TADS are actually positioned within a global chromosomal context.

In addition to the valuable visualizations, 4DMax accurately predicts chromosome position at timepoints where data is excluded from the 4DMax algorithm. The interpolated maps from 4DMax frequently show higher similarity to true contact maps at their corresponding time than to true contact maps at adjacent times presented to the model. This is particularly promising because it indicates the high similarity of retrieved biological features is not a product of low chromosomal structural change in temporal segments of the time series, but rather that novel inferences are being made to the actual position of the chromosome at times where no hi-c data is available. Given these findings 4DMax could be used by other labs as a preliminary substitute for expensive $\mathrm{Hi}-\mathrm{C}$ experiments when examining a genomic process over time.

4DMax is easily integrated into any time series Hi-C pipeline. Our model stability experiments show computational stability to variation of parameters such as contact map resolution and granularity while maintaining a sufficiently short run time. The structures generated by 4DMax show high correlation to input contact matrices and the synthetic contact maps derived from predicted 4DMax structures frequently have high correlation with real contact maps, even at times where no contact map data is presented to the model. 4DMax derived contact maps retain biologically relevant higher order features such as $A B$ compartment and TAD placement.

Despite these promising results the time scale of all real $\mathrm{Hi}-\mathrm{C}$ datasets tested is in the order of days, therefore it is possible that significant changes to chromosome conformation may occur at smaller intervals not captured by existing data. To address this concern in the future 4DMax will have to be applied to future time series $\mathrm{Hi}-\mathrm{C}$ datasets with smaller time intervals and additional assays for validation of conformation such as Capture $\mathrm{Hi}-\mathrm{C}$ and microscopy data. 


\section{Methods}

\section{Description of 4DMax algorithm}

4DMax is intended for researchers interested in inspecting the changing structural conformation of the genome over the duration of a dynamic biological process. We assume that the biological process occurs over a time interval $I=(0, T)$. We represent a chromosomes movement over a time interval as a collection of $n$ points in 4D space. Let $S$ be a 4D chromosome structure. $S=S\{t\} t \in I$, where $S\{t\}=\left\{S_{i}\{t\}\right\}$ and $S_{i}\{t\} \in R^{3} x I$, where $S_{\mathrm{i}}\{t\}$ denotes the $x, y, z$ coordinate of the $i^{\text {th }}$ bin of a chromosome at time $t$.

We view the chromosome structure $S$ as a structure in 4-dimensional space (3 spatial, 1 time), denoted $S \in\left(R^{3} X I\right)$. We use $S\{t\}$ to denote the structure's spatial position at a given time.

\section{Maximum likelihood}

We use a likelihood function as a loss function to compute chromosome conformation from the contact maps. The likelihood of structural conformation $\mathrm{S}$ can be modeled as the product of the probability of the observed HiC contact maps $H=\{H\}$ conditioned on $S\{t\}$

$$
L(S)=\prod_{t} P(H \mid S\{t\})
$$

$P(H \mid S\{t\})$ can be modeled as the product of individual distances in $H$ conditioned on $\mathrm{S}$ by assuming each constraint is independent. By assuming that each constraint $H_{i} \in H$ is conditionally independent of other constraints we rewrite the likelihood as

$$
L(S)=\prod_{t} \prod_{i} P\left(H_{i} \mid S\{t\}\right)
$$

Because our Hi-C samples were taken during some point during the biological process being observed, we know $\mathbb{T} \in I$, however, if we select a high granularity of $I$ there are certain $t \in I$ such that $t \notin \mathbb{T}$. Thus we can separate our $L(S)$ by

$$
L(S)=\prod_{t \in \mathbb{T}} \prod_{i} P\left(H_{i} \mid S\{t\}\right) * \prod_{t \notin \mathbb{T}} \prod_{i} P\left(H_{i} \mid S\{t\}\right)
$$

Because the logarithmic function is monotone we can take the logarithm of $L(S)$ without the argmax changing, yielding 


$$
L(S)=\sum_{t \in \mathbb{T}} \log \left(\prod_{i} P\left(H_{i} \mid S\{t\}\right)\right)+\sum_{t \notin \mathbb{T}} \log \left(\prod_{i}\left(P\left(H_{i} \mid S\{t\}\right)\right)\right.
$$

When $t \in \mathbb{T}$ we assume that observed contact maps are drawn from a gaussian probability distribution

$$
P\left(H_{i} \mid S\{t\}\right)=P\left(H_{i}\{t\} \mid S\{t\}\right) \sim \frac{1}{\sigma \sqrt{2 \pi}} \exp \left(\frac{-1}{2 \sigma^{2}}\left(D_{i}\{t\}-H_{i}\{t\}\right)^{2}\right)
$$

where $D_{i}\{t\}$ is the actual euclidean distance between the pair of regions index by $i$, computed from $(x, y, z)$ coordinates $\sigma$ is the standard deviation of the gaussian distribution. By assumption of normal distribution we know

$$
\sigma=\sqrt{\frac{\sum_{i}\left(H_{i}-D_{i}\right)^{2}}{n}}
$$

Using algebra we can manipulate equation (5) to resemble a component of the first right hand summation term in equation (4) as shown in (7).

$$
\prod_{i} P\left(H_{i} \mid S\right)=\left(\frac{1}{\sigma \sqrt{2 \pi}}\right)^{n} * \exp \left(\frac{-1}{2 \sigma^{2}} \sum_{i}^{n}\left(D_{i}\{t\}-H_{i}\{t\}\right)^{2}\right)
$$

Thus, by taking the logarithm of both sides of (7) we obtain

$$
\log \left(\prod_{i} P\left(H_{i} \mid S\right)\right)=-\frac{\sum_{i}\left(H_{i}\{t\}-D_{i}\{t\}\right)^{2}}{2 \sigma^{2}}-n * \log (\sigma)
$$

We can substitute equation (6) into equation (6) to remove all dependence on $\sigma$ and obtain

$$
\log \left(\prod_{i} P\left(H_{i} \mid S\right)\right)=\frac{n}{2}-n * \log \left(\sqrt{\frac{\sum\left(H_{i}\{t\}-D_{i}\{t\}\right)^{2}}{n}}\right)
$$

When $t \notin \mathbb{T}$ we define

$$
\begin{aligned}
& a_{1}(t)=\max \tau \in S_{\tau} \text { where } \tau<t \\
& a_{2}(t)=\min \tau \in S_{\tau} \text { where } \tau>t \\
& w_{1}(t)=\frac{t-a_{2}(t)}{\left|a_{1}(t)-a_{2}(t)\right|}, \quad w_{2}(t)=1-w_{1}(t)
\end{aligned}
$$

And assume

$$
P\left(H_{i} \mid S\{t\}\right)=w_{1}(t) * P\left(H_{i}\left\{a_{1}(t)\right\} \mid S\{t\}\right)+w_{2}(t) * P\left(H_{i}\left\{a_{2}(t)\right\} \mid S\{t\}\right) .
$$

Because $a_{1}(t), a_{2}(t) \in \mathbb{T}$ we use substitution with equation (5) and (6) to obtain. 


$$
\begin{gathered}
\text { When } t \notin \mathbb{T}, \quad \log \left(\prod_{i} P\left(H_{i} \mid S(t)\right)\right)= \\
\frac{n}{2}-n *\left[w_{1}(t) * \log \left(\sqrt{\frac{\sum_{i}\left(H_{i}\left\{a_{1}(t)\right\}-D_{i}\{t\}\right)^{2}}{n}}\right)+w_{2}(t) * \log \left(\sqrt{\frac{\sum_{i}\left(H_{i}\left\{a_{2}(t)\right\}-D_{i}\{t\}\right)^{2}}{n}}\right)\right] .
\end{gathered}
$$

By substituting equation (9) and (11) into the $t \in \mathbb{T}$ and $t \notin \mathbb{T}$ terms of (4) we obtain a well defined likelihood function (13).

$$
\begin{gathered}
L(S)=-\prod_{t \in \mathbb{T}} \prod_{i} \log \frac{n}{2}-n * \log \left(\sqrt{\frac{\sum\left(H_{i}\{t\}-D_{i}\{t\}\right)^{2}}{n}}\right) * \prod_{t \notin \mathbb{T}} \prod_{i} \frac{n}{2}-n * \\
{\left[w_{1}(t) * \log \left(\sqrt{\frac{\sum_{i}\left(H_{i}\left\{a_{1}(t)\right\}-D_{i}\{t\}\right)^{2}}{n}}\right)+w_{2}(t) * \log \left(\sqrt{\frac{\sum_{i}\left(H_{i}\left\{a_{2}(t)\right\}-D_{i}\{t\}\right)^{2}}{n}}\right)\right]}
\end{gathered}
$$

To obtain the likelihood loss function we just take the negation of (13) to get

$$
D_{\text {likelihood }}(S)=-L(S)
$$

\section{Distance Function:}

Because the purpose of 4DMax is to represent structural changes in time as a continuous evolution, rather than provide individual snapshot images, it is important the motion between frames be fluid. To help ensure this we include a penalizing term, distance loss

$$
\begin{aligned}
D(s) & =\sum_{t} \delta(s(t)) \\
& =\sum_{t} \sum_{i} \delta\left(s_{i}\{t\}, s_{i}\{t+1\}\right) \\
& =\sum_{t} \sum_{i} \sqrt{\sum_{c}\left(s_{i, c}\{t\}-s_{i, c}\{t+1\}\right)^{2}} \quad c \in\{x, y, z\} .
\end{aligned}
$$

This minimizes jumps between frames and results in more continuous structures.

\section{Optimization}

We optimize our structures coordinates by constructing a linear combination of our distance loss function and likelihood-loss function and incrementally adjusting via gradient ascent, yielding 


$$
S\{t\}_{x, y, z}=S\{t\}_{x, y, z}+\lambda *\left(-D_{\text {likelihood }}(S)+\left(\eta *\left(D_{\text {movement }}(S)\right)\right)\right.
$$

Where $\eta$ is a weighing constant and $\lambda$ is the learning rate.

Unless stated otherwise we run experiments with $\eta=1000$ and $\lambda=0.0001$.

\section{Interpolation of Contacts}

We interpolate contacts by first running 4DMax excluding input $\mathrm{Hi}-\mathrm{C}$ maps at time $t$ of interest. From the 4D model we extract the predicted 3D structure at time $t$. Using this 3D mode we assume an inverse relationship between spatial distance and contact frequency as described in Oluwadare $2019{ }^{6}$ with map generation equation $I F_{i, j}=d_{i, j}^{\gamma}$ we use $\gamma$ values of $-1$.

\section{AB Compartment Analysis}

$A B$ compartments are identified in the manner outlined by Lieberman $2009^{1}$. We first obtain observed over expected (O/E) matrices for contact maps, where expected values are the mean contact frequency between bins of a given distance. From O/E matrices we treat rows as vectors and obtain pearson correlation matrices. From the correlation matrices we perform principal component analysis (PCA). We assign compartments to each bin based on the sign of its corresponding row's PC1 value. Trajectories are obtained by performing PCA on AB compartment sign assignment vectors. Scatterplots are obtained by mapping pc1 values between two corresponding $A B$ profiles as $(x, y)$ coordinates.

\section{TAD Identification}

TADs were identified using the directionality index approach ${ }^{4}$ as implemented by HiCtool ${ }^{14}$. This procedure involves the identification of a directionality index. Using the directionality index a hidden markov model (HMM) is used to identify biased states via the viterbi algorithm. From the HMM emissions TAD coordinates are derived as consecutive downstream bias states ${ }^{14}$. We compare TAD profiles of different contact maps using percent overlap (PO). We consider a TAD boundary from one profile overlapping if it occurs within .5Mb of a same direction TAD boundary on the compared profile. ${ }^{14}$

\section{Statistical analysis}

Pearson correlation coefficient (PCC) and Spearman correlation coefficient (SPC) were used for evaluating similarity of contact matrices and distance vectors of 3D structures. Comparison between 4D structures were based on average correlation between 3D structures at each corresponding time point. 


\section{Data availability}

All Hi-C data were downloaded from the Gene Expression Omnibus (GEO). Cardiomyocyte data was found at accession number GSE $106690^{8}$ and induced pluripotency data was found at the accession number GSE966117.

\section{Code Availability}

4DMax was built using python. 4DMax as well as the software for all enclosed experiments is available at https://github.com/Max-Highsmith/4DMax.

\section{References}

1. Lieberman-Aiden, E. et al. Comprehensive mapping of long-range interactions reveals folding principles of the human genome. Science 326, 289-293 (2009).

2. Lajoie, B. R., Dekker, J. \& Kaplan, N. The Hitchhiker's guide to Hi-C analysis: practical guidelines. Methods 72, 65-75 (2015).

3. van Steensel, B. \& Dekker, J. Genomics tools for unraveling chromosome architecture. Nat. Biotechnol. 28, 1089-1095 (2010).

4. Nora, E. P. et al. Spatial partitioning of the regulatory landscape of the X-inactivation centre. Nature 485, 381-385 (2012).

5. Dixon, J. R. et al. Topological domains in mammalian genomes identified by analysis of chromatin interactions. Nature vol. 485 376-380 (2012).

6. Oluwadare, O., Highsmith, M. \& Cheng, J. An Overview of Methods for Reconstructing 3-D Chromosome and Genome Structures from Hi-C Data. Biol. Proced. Online 21, 7 (2019).

7. Stadhouders, R. et al. Transcription factors orchestrate dynamic interplay between genome topology and gene regulation during cell reprogramming. Nat. Genet. 50, 238-249 (2018).

8. Bertero, A. et al. Dynamics of genome reorganization during human cardiogenesis reveal 
an RBM20-dependent splicing factory. Nat. Commun. 10, 1538 (2019).

9. Marti-Renom, M. A. et al. Challenges and guidelines toward 4D nucleome data and model standards. Nat. Genet. 50, 1352-1358 (2018).

10. Di Stefano, M. et al. Transcriptional activation during cell reprogramming correlates with the formation of 3D open chromatin hubs. Nat. Commun. 11, 2564 (2020).

11. Gradient Ascent. Independent Component Analysis (2004) doi:10.7551/mitpress/3717.003.0016.

12. Rossi, R. J. Mathematical Statistics: An Introduction to Likelihood Based Inference. (John Wiley \& Sons, 2018).

13. Oluwadare, O., Zhang, Y. \& Cheng, J. A maximum likelihood algorithm for reconstructing 3D structures of human chromosomes from chromosomal contact data. BMC Genomics 19, $161(2018)$

14. Calandrelli, R., Wu, Q., Guan, J. \& Zhong, S. GITAR: An Open Source Tool for Analysis and Visualization of Hi-C Data. Genomics Proteomics Bioinformatics 16, 365-372 (2018). 


\section{Figures}

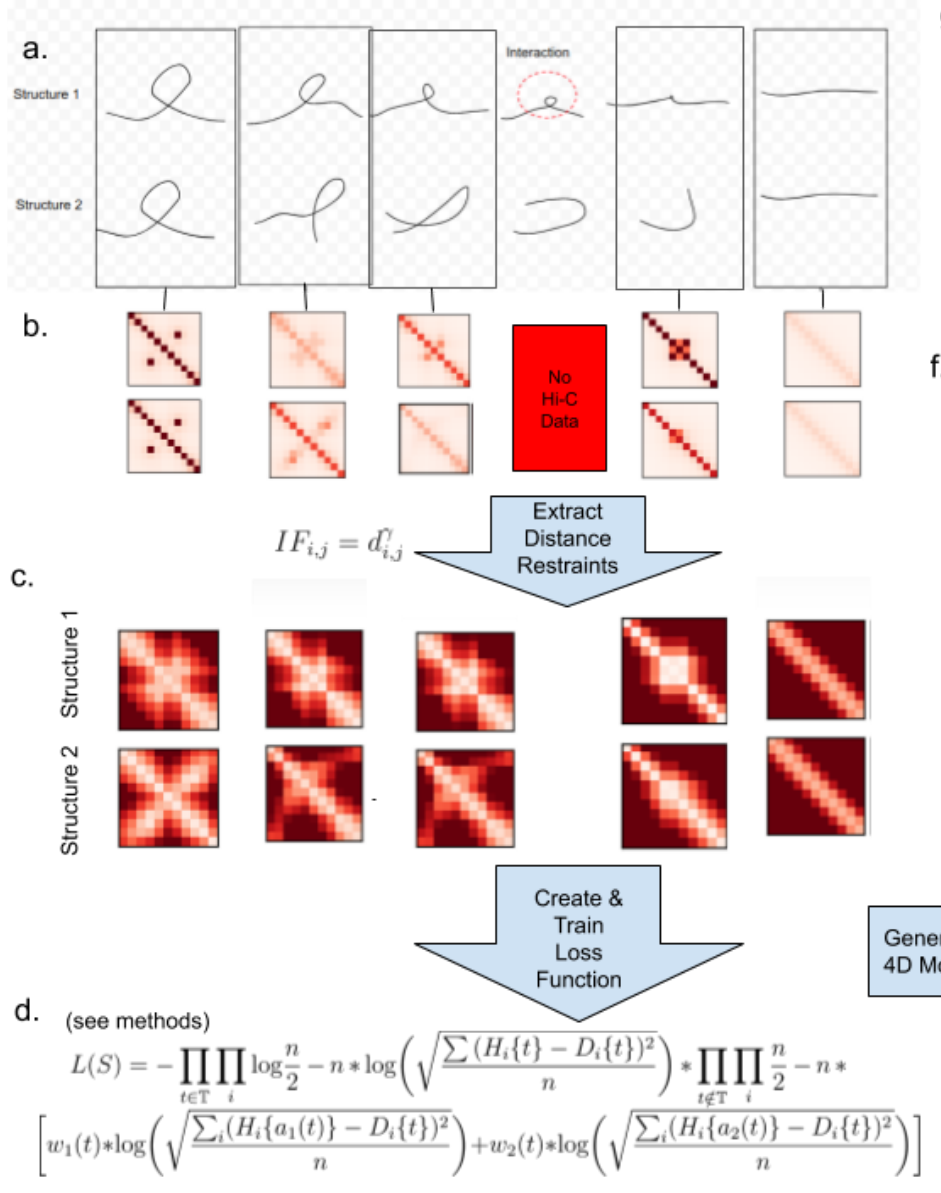

g.
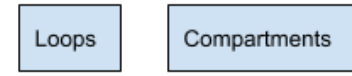

TADs

f.

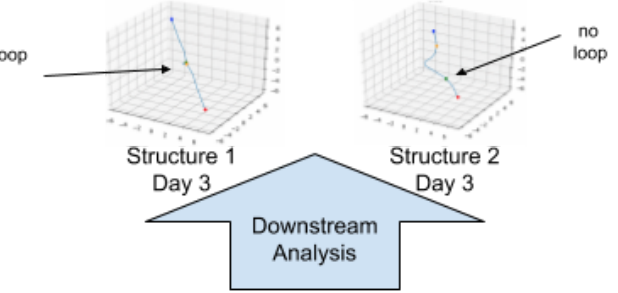

e.

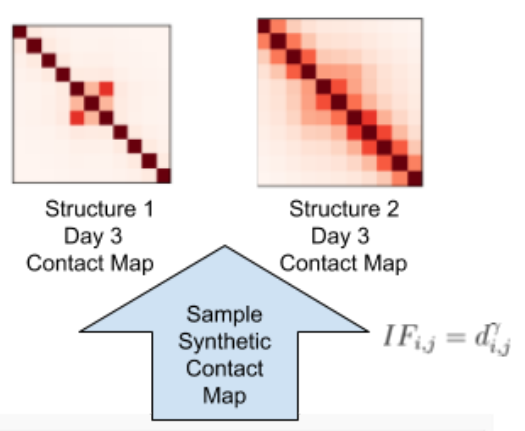

Map

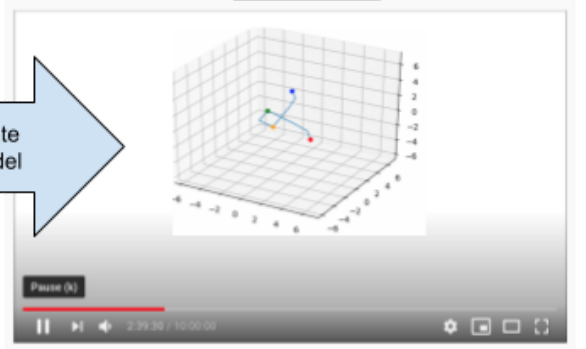

40 Model

\section{Figure 1: Overview of 4DMax approach}

Graphic elucidates the 4DMax workflow using a simplified synthetic dataset as illustration. (a) Drawings of two potential chromosomal trajectories from identical starting and ending conformations. A significant contact at center exists in structure 1 but not structure 2. (b) Contact maps obtained through synthetic Hi-C experiments on each day in process. (c) Distance restraints derived from available contact maps. (d) Likelihood function for predicting 4D conformation. (e) Video of changing chromosome conformation. (f) Synthetic contact maps extracted at time of interest (g) Different 3D structural conformations on day 3. 
a.

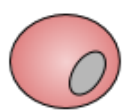

D0

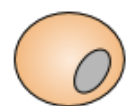

D2

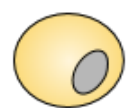

D4

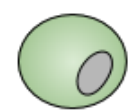

D6

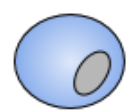

D8

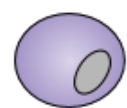

PSC
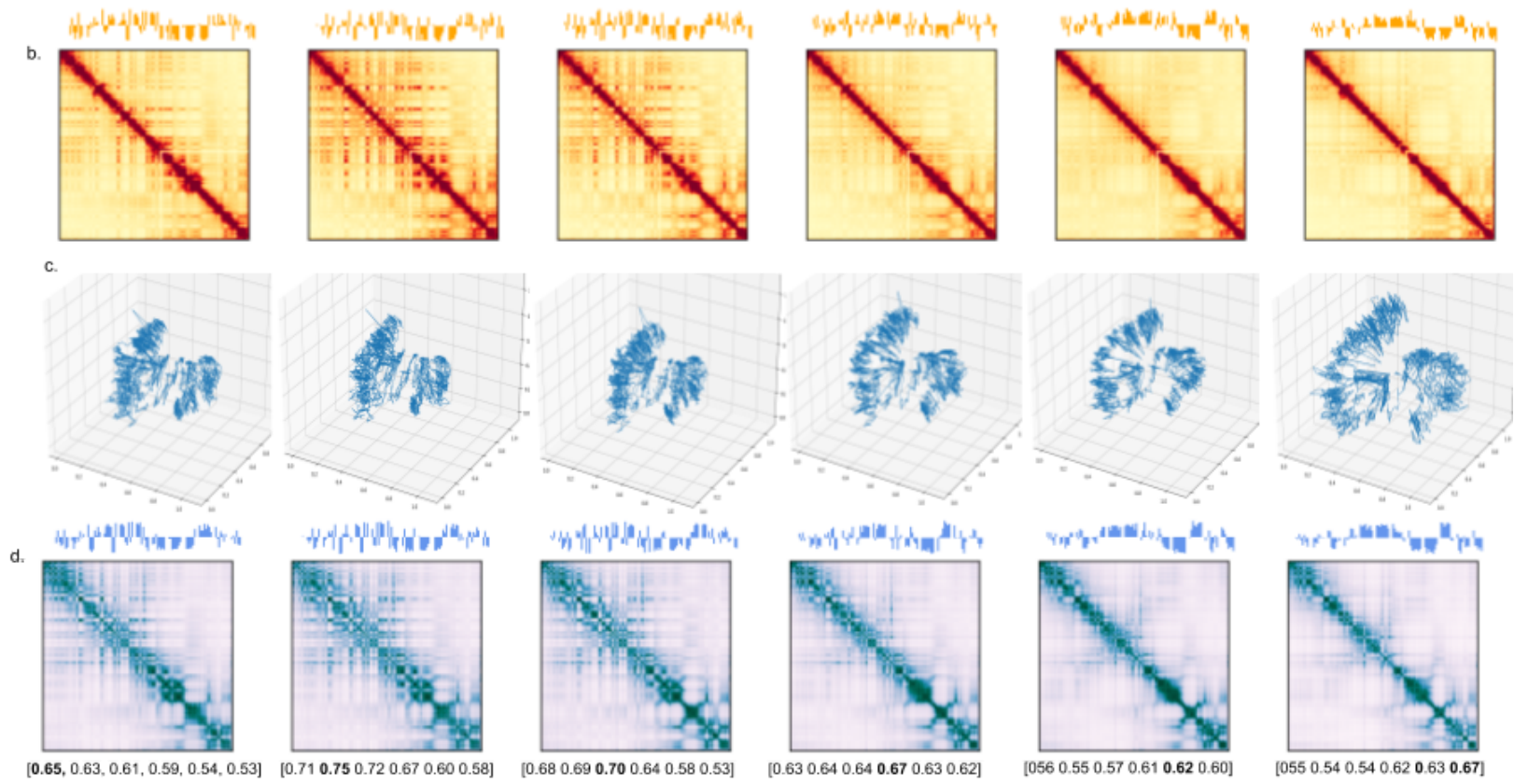

Figure 2: Simulation of 4DMax Structures

Diagram of Outputs. (a) Outline of the different stages of iPSC dataset. (b) Contact map of chromosome 13 by time, AB compartment vector shown above map. (c) 4DMax prediction of structural conformation of chromosome 13 at time. (d) Reconstructed contact map using simulated Hi-C of 4DMax structure, number below indicate spearman correlation between above reconstructed contact map and real contact maps at each time point. 
a.
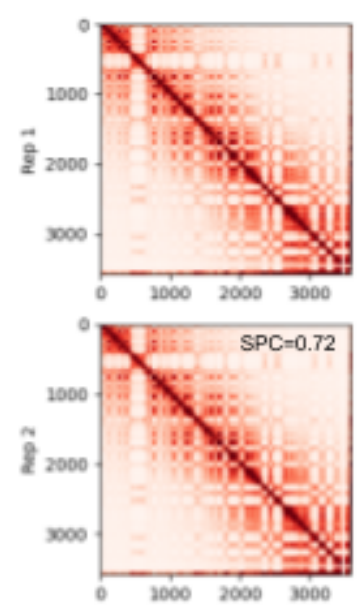

Real Hi-C
Mouse iPSC Day 2 Chro 2
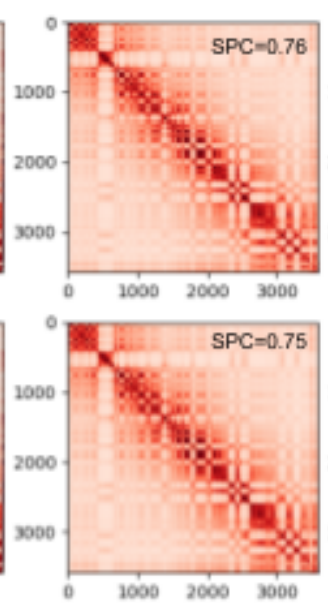

Reconstruction
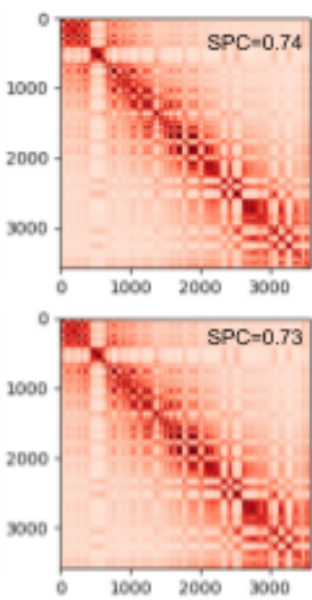

Interpolation

b.

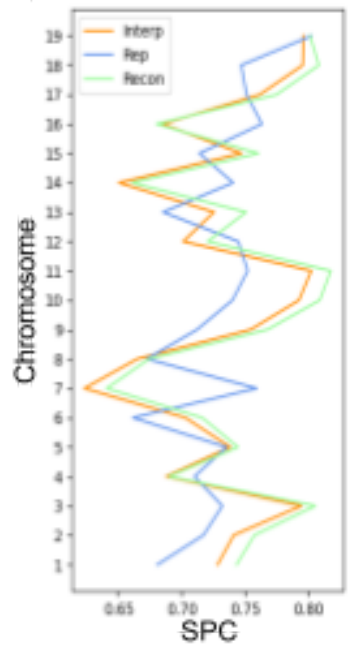

Human Cardiomyocyte Day 2 Chro 7
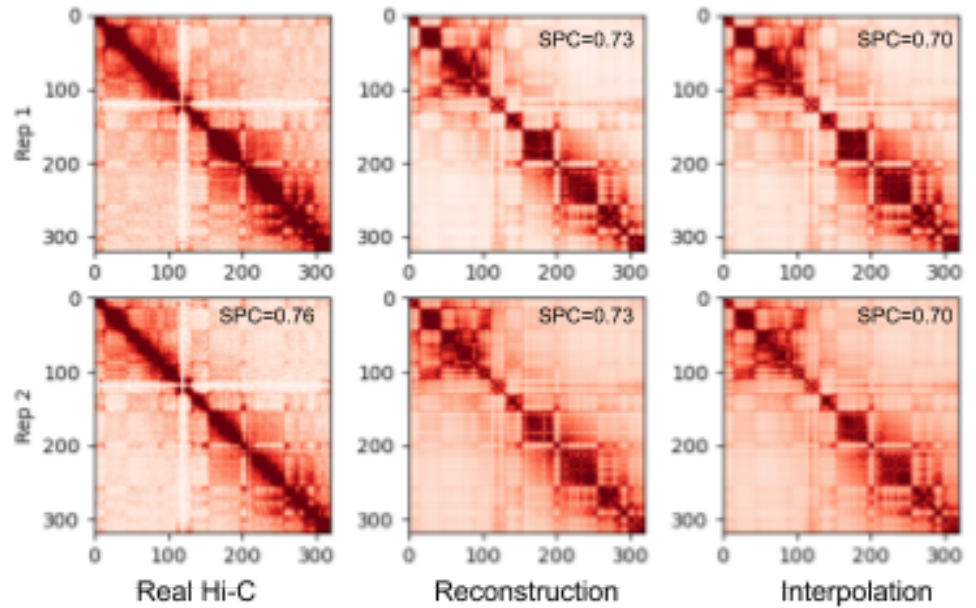

\section{Figure 3: Predictions of Hi-C Contact Maps}

Example contact map comparison. (a) Contact maps of iPSC on day 2 chromosome 2 from Real Hi-C, 4DMax reconstruction and 4DMax day 2 agnostic interpolation model. (b) SPC of iPSC contact maps relative to Real Hi-C for each chromosome on day 2. (c) Contact maps of cardiomyocyte data on day 2 chromosome 7 from Real Hi-C, 4DMax reconstruction and 4DMax day 2 agnostic interpolation model. (d) SPC of cardiomyocyte contact maps to Real Hi-C for each chromosome on day 2. 
a.

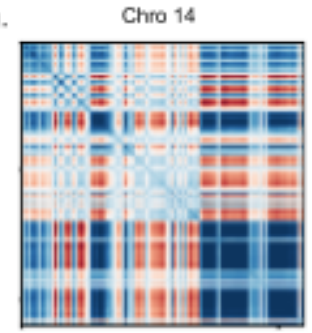

Real Hi-C

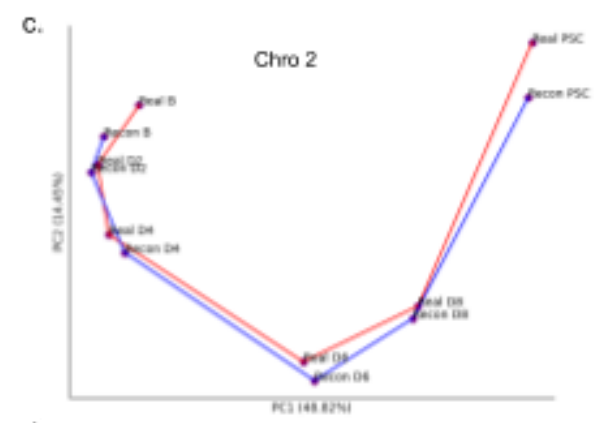

d.

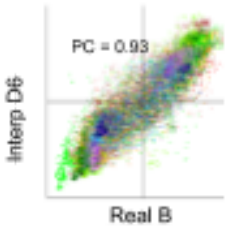

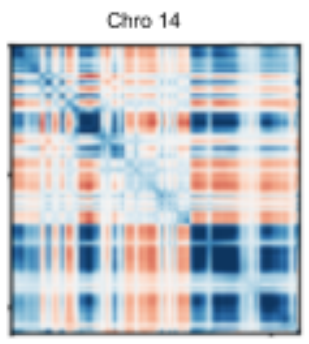

Recon Hi-C

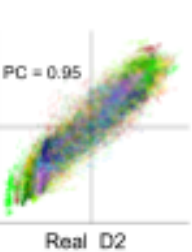

b.

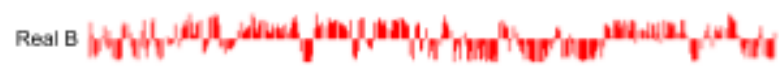

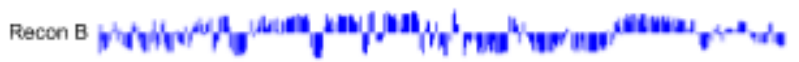

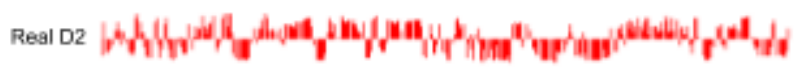

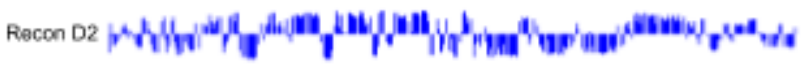
Real 04 , thin

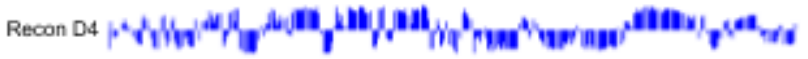

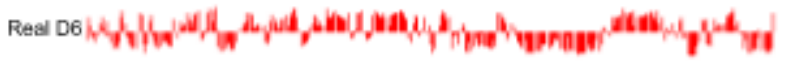

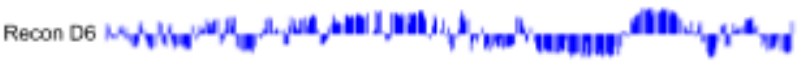

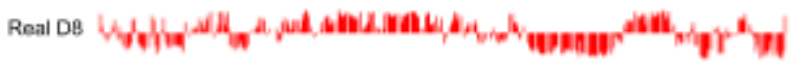

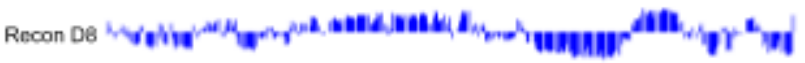

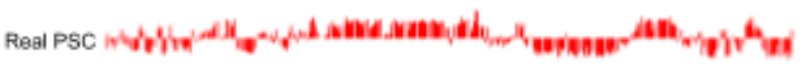

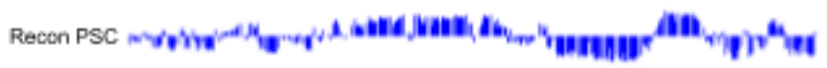

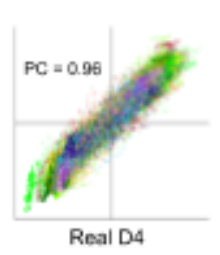

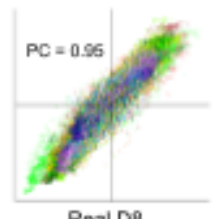

Real DQ

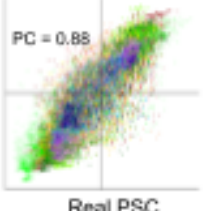

Real PSC

Figure 4: $A B$ compartment analysis

Analysis of $A B$ compartment features of 4DMax generated contact maps. (a) Pearson correlation matrices of chromosome 14 day 2 using Real $\mathrm{Hi}-\mathrm{C}$ and synthetic contact maps obtained from the 4DMax model. (b) AB compartment vectors from chromosome 14 (red) real $\mathrm{Hi}-\mathrm{C}$ data (blue) synthetic contact maps obtained from 4DMax model. (c) Trajectory curve of two largest principal components (red) real Hi-C (Blue) Reconstructed Hi-C. (d) Scatter plot of $100 \mathrm{~kb}$ binned $A B$ compartment vectors where $x$ value is bins Real Data $P C 1$ value and $y$ value is interpolated Contact maps PC1 value. 

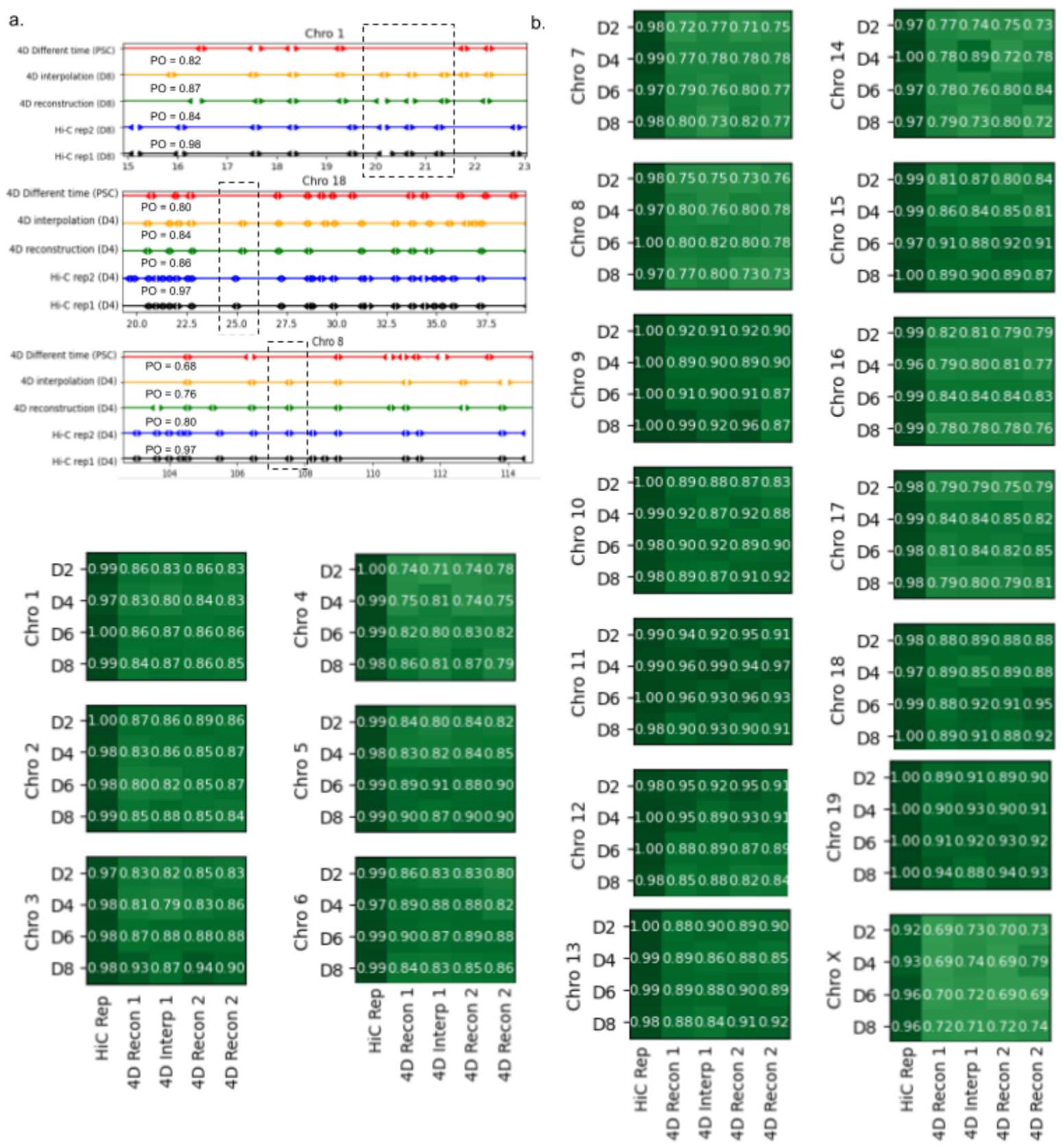

\section{Figure 5: Topologically Associated domain analysis}

HiCtool identified topologically associated domains. (a) Select images of TAD boundaries on (black) Real Hi-C replicate 1, (blue) Real Hi-C replicate 2, (green) 4DMax Reconstructed Map, (orange) 4DMax Interpolated Hi-C Map and 4DMax Recon Map at a different time point. PO metric quantifies the percent of TAD boundaries found within $0.5 \mathrm{Mb}$ of a boundary identified in $\mathrm{Hi}-\mathrm{C}$ rep1. (b) PO of Interpolated and Reconstructed 4DMax TAD positions for both replicates across all chromosomes. 
bioRxiv preprint doi: https://doi.org/10.1101/2020.11.10.377002; this version posted November 11, 2020. The copyright holder for this preprint (which was not certified by peer review) is the author/funder, who has granted bioRxiv a license to display the preprint in perpetuity. It is made available under aCC-BY 4.0 International license. 

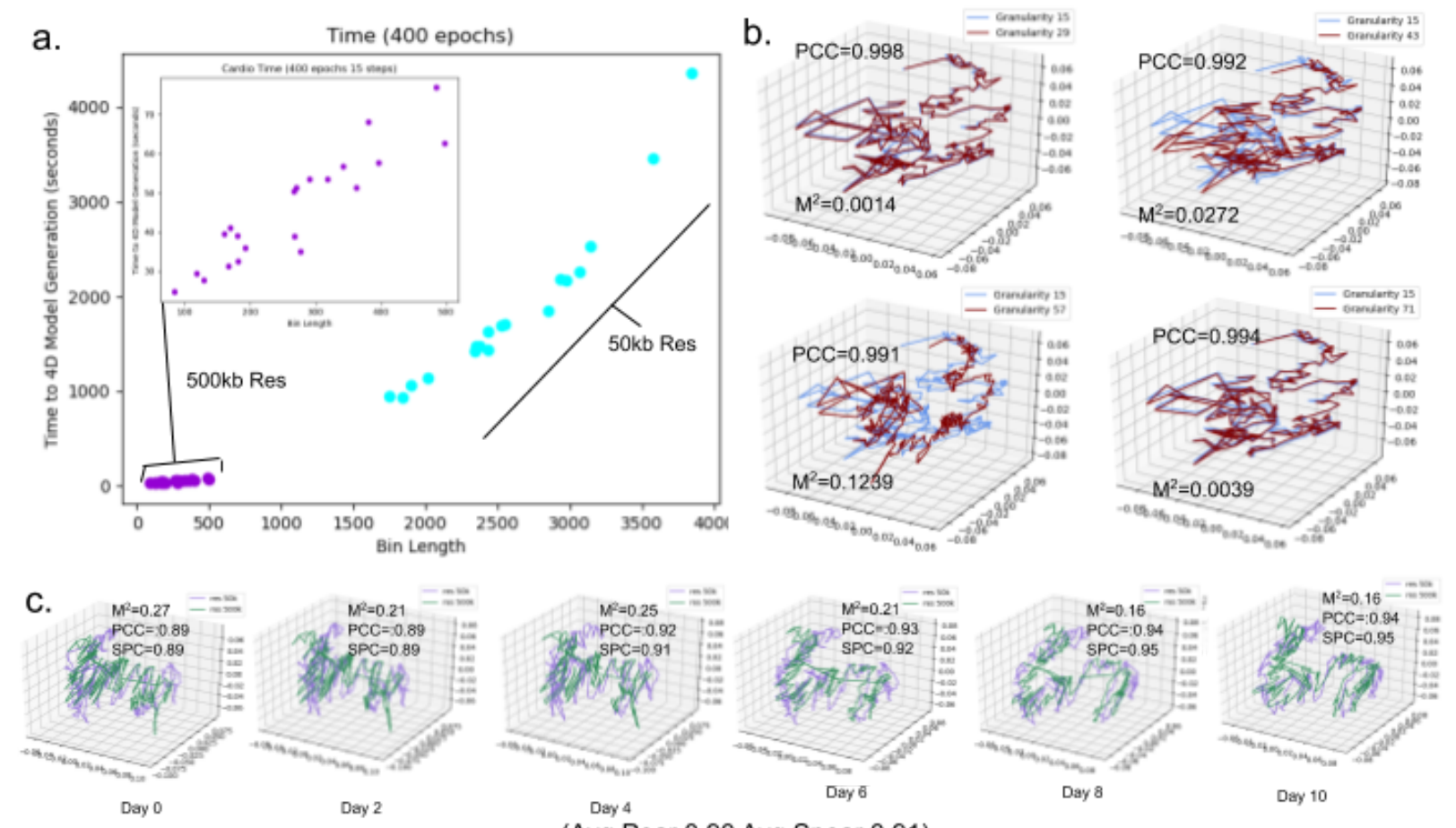

(Avg Pear 0.90 Avg Spear 0.91)

\section{Figure 6: 4DMax Computational Evaluation}

Evaluation of runtimes and computational stability. (a) Scatter plot of chromosome bin lengths and time to completion using 400 epoch (purple) 500kb resolution chromosome and (blue) 50 $\mathrm{kb}$ resolution chromosomes. (b) 3D plot of predicted cardiomyocyte chromosome 10 on day 5 with varying granularity values. Spearman correlation Mean Squared distance compares (blue) granularity 15 structure to higher granularity structures (red). (c) 3D plots comparing (purple) 50 $\mathrm{kb}$ resolution chromosome 1 to (green) 500kb resolution iPSC chromosome 1 on each day in time series. 


\section{Acknowledgements}

This project is partially supported by two NSF grants (no. IOS1545780 and no. DBI1149224).

\section{Author Contributions}

$\mathrm{MH}$ and $\mathrm{JC}$ conceived the project. $\mathrm{MH}$ performed all experiments and drafted the manuscript. $\mathrm{JC}$ revised the manuscript.

\section{Competing Interests}

The authors declare no competing interests

\section{Additional Information}

Supplementary information is available for this paper at https://docs.google.com/document/d/1A08wOei5grKH7A3YjdAcGE3ScY9mvoxpqeC03Nm1olQ/ edit\# 


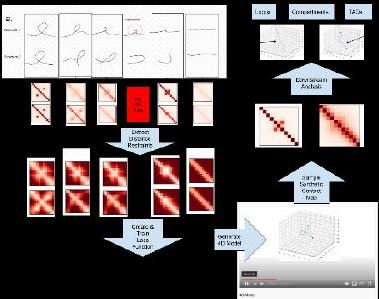




\section{0}
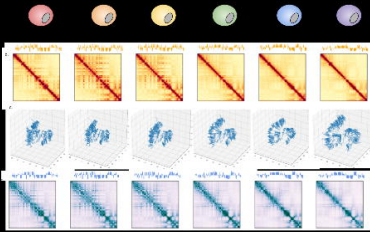

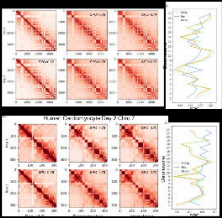


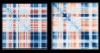

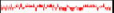

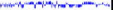

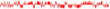

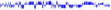

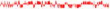

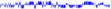

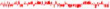

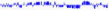

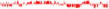

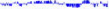

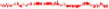

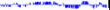
1

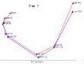

-
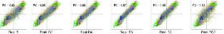


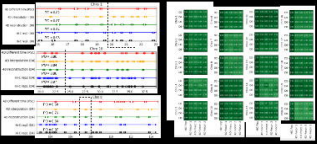


a.

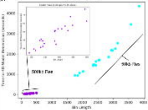

b.

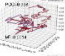

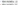

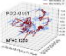

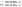

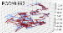

H-tos:

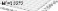
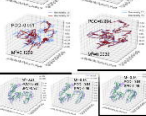

t $+2 t$

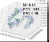

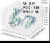

